\title{
Well-established nucleon resonances revisited by double-polarization measurements
}

\author{
A. Thiel, ${ }^{1}$ A.V. Anisovich, ${ }^{1,2}$ D. Bayadilov, ${ }^{1,2}$ B. Bantes,${ }^{3}$ R. Beck,${ }^{1}$ Yu. Beloglazov, ${ }^{1,2}$ M. Bichow, ${ }^{4}$ S. \\ Böse, ${ }^{1,5}$ K.-Th. Brinkmann, ${ }^{1,5}$ Th. Challand, ${ }^{6}$ V. Crede, ${ }^{7}$ F. Dietz, ${ }^{5}$ P. Drexler, ${ }^{5}$ H. Dutz, ${ }^{3}$ H. Eberhardt, ${ }^{3}$ \\ D. Elsner, ${ }^{3}$ R. Ewald, ${ }^{3}$ K. Fornet-Ponse, ${ }^{3}$ St. Friedrich, ${ }^{5}$ F. Frommberger,${ }^{3}$ Ch. Funke, ${ }^{1}$ M. Gottschall, ${ }^{1}$ M. \\ Grüner, ${ }^{1}$ E. Gutz, ${ }^{1}$ Ch. Hammann, ${ }^{1}$ J. Hannappel, ${ }^{3}$ J. Hartmann, ${ }^{1}$ W. Hillert, ${ }^{3}$ Ph. Hoffmeister,${ }^{1}$ Ch. Honisch, ${ }^{1}$ \\ I. Jaegle, ${ }^{6}$ I. Jürgensen, ${ }^{1}$ D. Kaiser ${ }^{1}$ H. Kalinowsky, ${ }^{1}$ F. Kalischewski, ${ }^{1}$ S. Kammer, ${ }^{3}$ I. Keshelashvili, ${ }^{6}$ \\ V.Kleber, ${ }^{3}$ F. Klein, ${ }^{3}$ E. Klempt, ${ }^{1}$ B. Krusche,${ }^{6}$ M. Lang, ${ }^{1}$ I. Lopatin, ${ }^{2}$ Y. Maghrbi, ${ }^{6}$ K. Makonyi, ${ }^{5}$ V . \\ Metag, ${ }^{5}$ W. Meyer, ${ }^{4}$ J. Müller, ${ }^{1}$ M. Nanova, ${ }^{5}$ V. Nikonov, ${ }^{1,2}$ R. Novotny, ${ }^{5}$ D. Piontek, ${ }^{1}$ G. Reicherz, ${ }^{4}$ \\ A. Sarantsev, ${ }^{1,2}$ Ch.Schmidt, ${ }^{1}$ H. Schmieden, ${ }^{3}$ T.Seifen, ${ }^{1}$ V.Sokhoyan, ${ }^{1}$ V.Sumachev, ${ }^{2}$ U. Thoma, ${ }^{1}$ \\ H. van Pee, ${ }^{1}$ D. Walther ${ }^{1}$ Ch. Wendel,${ }^{1}$ U. Wiedner ${ }^{4}$ A. Wilson, ${ }^{7}$ A. Winnebeck, ${ }^{1}$ and Y. Wunderlich ${ }^{1}$ \\ (The CBELSA/TAPS Collaboration) \\ ${ }^{1}$ Helmholtz-Institut für Strahlen- und Kernphysik, Universität Bonn, Germany \\ ${ }^{2}$ Petersburg Nuclear Physics Institute, Gatchina, Russia \\ ${ }^{3}$ Physikalisches Institut, Universität Bonn, Germany \\ ${ }^{4}$ Institut für Experimentalphysik I, Universität Bochum, Germany \\ ${ }^{5}$ II. Physikalisches Institut, Universität Gießen, Germany \\ ${ }^{6}$ Physikalisches Institut, Universität Basel, Switzerland \\ ${ }^{7}$ Department of Physics, Florida State University, Tallahassee, USA
}

(Dated: February 12, 2018)

\begin{abstract}
The first measurement is reported of the double-polarization observable $G$ in photoproduction of neutral pions off protons, covering the photon energy range from 620 to $1120 \mathrm{MeV}$ and the full solid angle. $G$ describes the correlation between the photon polarization plane and the scattering plane for protons polarized along the direction of the incoming photon. The observable is highly sensitive to contributions from baryon resonances. The new results are compared to the predictions from SAID, MAID, and BnGa partial wave analyses. In spite of the long-lasting efforts to understand $\gamma p \rightarrow p \pi^{0}$ as the simplest photoproduction reaction, surprisingly large differences between the new data and the latest predictions are observed which are traced to different contributions of the $N(1535)$ with spin-parity $J^{P}=1 / 2^{-}$and $N(1520)$ with $J^{P}=3 / 2^{-}$. In the third resonance region, where $N(1680)$ with $J^{P}=5 / 2^{+}$production dominates, the new data are reasonably close to the predictions.
\end{abstract}

PACS numbers: 14.20

Symmetry arguments have led Gell-Mann and Zweig to introduce the concept of quarks as constituents of mesons and baryons [1, 2]. Later it was realized that quarks carry a new kind of charge, color, and are the sources of gluons transmitting the binding forces. A new theory evolved, quantum chromodynamics [3], which proved to be very successful for large momenta where the interaction becomes weaker. In the region of meson and baryon resonances, QCD resists any perturbative approach, and numerical calculations on a space-time lattice are necessary. It has been only recently that the full baryon spectrum including physical quantum numbers was presented [4], even though unrealistically large quark masses had to be used. Qualitatively, these lattice results confirm the classic quark model calculations [5], whereas the agreement with a fully relativistic model is less convincing [6]. Yet the experimental mass spectrum exhibits some remarkable differences to these calculations. The $N(1440)$ resonance with spin-parity $J^{P}=1 / 2^{+}$has a mass of $1440 \mathrm{MeV}$ and falls below its spin-parity partner $N(1535)$ with $J^{P}=1 / 2^{-}$while QCD on the lattice and quark model calculations predict the reverse. Also, above a mass of $1800 \mathrm{MeV}$, the number of observed resonances falls short of the number of predicted resonances.

Baryon resonances have very short life times, hence many resonances with different spin-parities overlap; their properties have to be unfolded from data in partial wave analyses. Most partial wave analyses are done using $\pi N$ elastic and charge exchange scattering, but photoproduction experiments offer the opportunity of analyzing resonances which were not accessible up to now. However, the number of independent observables necessary for a unique partial wave solution increases. Below the two-pion production threshold at $E_{\gamma}=310 \mathrm{MeV}$, the Watson theorem [7] relates the $\pi N$ phases to those in photoproduction of single pions. In this case the measurement of the differential cross section $d \sigma$ and of the photon beam asymmetry $\Sigma$ is sufficient to determine precisely the s- and p-wave multipoles $\left(l_{\pi}=0,1\right)$, as well as the small ratio of electric quadrupole (E2) and magnetic dipole (M1) amplitudes in the $N \rightarrow \Delta(1232)$ transition [8, 9]. Above the two-pion threshold, but still in the mass range where nucleon resonances carry only one unit of intrinsic orbital angular momentum, five experiments are sufficient to construct the spin-helicity structure of the photoproduction amplitude [10, 11]. In general, the 

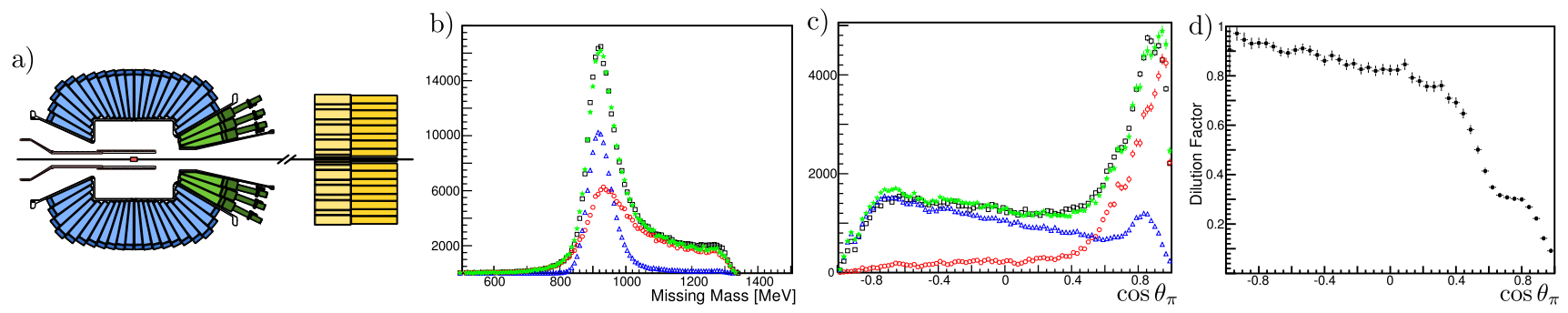

Figure 1. (Color online) a) The central part of the detector system. The CsI(Tl) crystals (blue and green) are read out via wavelength shifters and photodiodes or photomultipliers, $\mathrm{BaF}_{2}$ crystals (yellow) in forward direction with photomultipliers. b) The missing mass distribution, and (c) the $\pi^{0}$ angular distribution for reaction (1) for an incident photon energy of $E_{\gamma}=$ $1000 \pm 25 \mathrm{MeV}$; butanol $(\square)$, hydrogen $(\triangle)$, carbon $(\circ)$, and the sum of hydrogen and carbon data (*). From these distributions, the dilution factor $(\mathrm{d})$ is determined.

observation of eight polarization observables with high precision is required to construct the electric $(E)$ and magnetic $(M)$ multipoles governing the process $[12,13$.

In this letter we report on a first measurement of the double-polarization observable $G$ in photoproduction of neutral pions off protons covering the second and third resonance region. Linearly polarized photons were produced by scattering of a $3.2 \mathrm{GeV}$ electron beam [14] off a diamond crystal [15], whereby maximal polarization of $65 \%$ at $950 \mathrm{MeV}$ and $59 \%$ at $1150 \mathrm{MeV}$ for a second data set were reached. The photons then hit a butanol $\left(\mathrm{C}_{4} \mathrm{H}_{10} \mathrm{O}\right)$ target with longitudinally polarized protons [16], with a mean proton polarization of about $75 \%$. The butanol target was replaced by a hydrogen or carbon target for background studies and for normalization. The incoming photons may produce a $\pi^{0}$ in the reaction

$$
\vec{\gamma} \vec{p} \rightarrow p \pi^{0} .
$$

For linearly polarized photons (with polarization $\mathrm{p}_{\gamma}$ ) and protons (with polarization $\mathrm{p}_{\mathrm{T}}$ ), the number of events $N$ at the polar angle $\theta_{\pi}$ due to reaction (1) as a function of the azimuthal angle $\phi_{\pi}$ can be written in the form

$$
\frac{N\left(\phi_{\pi}, \theta_{\pi}\right)}{N_{0}\left(\theta_{\pi}\right)}=1-\mathrm{p}_{\gamma} \Sigma_{\mathrm{B}} \cos \left(2 \phi_{\pi}\right)+\mathrm{p}_{\gamma} \mathrm{p}_{\mathrm{T}} G_{\mathrm{B}} \sin \left(2 \phi_{\pi}\right)
$$

where $N_{0}$ is given by averaging $N\left(\phi_{\pi}, \theta_{\pi}\right)$ over $\phi_{\pi}$. The beam asymmetry $\Sigma_{\mathrm{B}}$ and the observable $G_{\mathrm{B}}$ for the butanol target are related to the corresponding quantities for scattering off free $(f)$ protons $\Sigma, G$ and bound $(b)$ nucleons by

$$
\Sigma_{\mathrm{B}}=\frac{N_{0}^{f} \Sigma+N_{0}^{b} \Sigma_{b}}{N_{0}^{f}+N_{0}^{b}} ; \quad G_{\mathrm{B}}=\frac{N_{0}^{f}}{N_{0}^{f}+N_{0}^{b}} \cdot G .
$$

The number of events in the denominator is the sum of the number of events where scattering took place off protons or a bound nucleon, $N_{0}=N_{0}^{f}+N_{0}^{b}$. There is no contribution of bound nucleons to $G_{\mathrm{B}}$ since carbon and oxygen nuclei carry no polarization. The quantity $D=N_{0}^{f} /\left(N_{0}^{f}+N_{0}^{b}\right)$ is called dilution factor and depends on the reaction and on the kinematical cuts performed.
It is also possible to determine $G$ without using the dilution factor by reversing the target polarization and regarding the difference $N^{+}\left(\phi_{\pi}, \theta_{\pi}\right)-N^{-}\left(\phi_{\pi}, \theta_{\pi}\right)$, given by $2 N_{0}^{f} \mathrm{p}_{\gamma} \mathrm{p}_{\mathrm{T}} G \sin \left(2 \phi_{\pi}\right)$. A similar equation can be written for a change of the photon polarization plane by $\pm \pi / 4$ 17.

Fig. 17 shows the calorimeter setup. Neutral pions are reconstructed from their decay into two photons and a measurement of their energy and direction in $\mathrm{CsI}(\mathrm{Tl})$ and $\mathrm{BaF}_{2}$ crystals enclosing the target hermetically. The proton direction is determined, assuming that it originated in the target center, from its hit in the surrounding three-layer scintillation fiber detector [18] or scintillation detectors in front of the crystals in forward direction, and its hit in the CsI or $\mathrm{BaF}_{2}$ crystals. In comparison to [19], two major instrumental changes have been introduced. The forward opening of the calorimeter is now covered by 90 additional CsI(Tl) crystals with photomultiplier readout in the main calorimeter and $216 \mathrm{BaF}_{2}$ crystals, $2.1 \mathrm{~m}$ downstream of the target. The tagger is now mounted horizontally. Beam electrons not producing bremsstrahlung are now deflected to the left and are stopped in a beam dump well behind the detector system.

In the first step of the reconstruction, events due to reaction (1) are selected using a series of kinematical cuts. Two classes of events are retained, events with three hits in the calorimeters and events with two hits. Both hits of a two-hit event are assumed to be photons; their invariant mass is calculated and required to fall into a $3 \sigma$ $( \pm 25 \mathrm{MeV})$ window centered at the $\pi^{0}$ mass. The proton is assumed to be stopped in some material, but due to energy and momentum conservation, the proton momentum can be reconstructed. If there is a third hit, one pair of hits is assumed to be photons, and it is checked that the pair forms a $\pi^{0}$, using again a $\pm 3 \sigma$ window. The third hit has to match the direction of the proton momentum reconstructed as missing momentum. Matching is defined by a cone, $\pm 7^{\circ}$ in the azimuthal and polar angles. Finally, a time coincidence is required between the tagger hit and hits in one of the scintillation detectors or 
a)

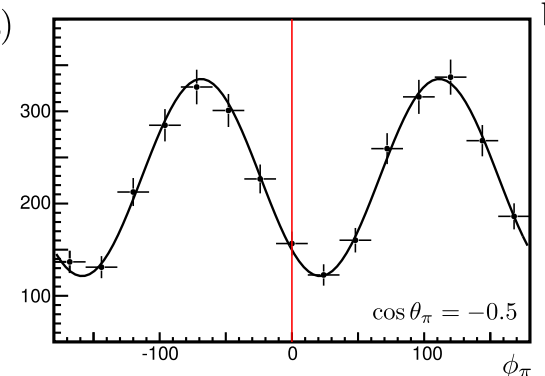

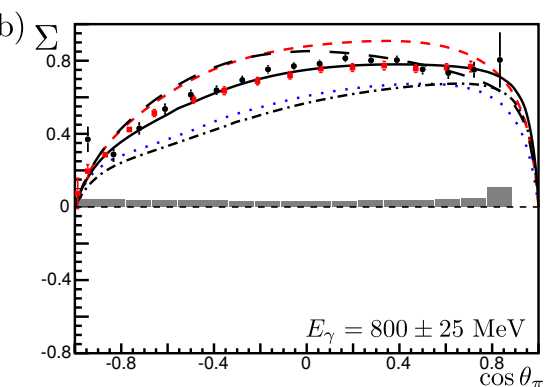

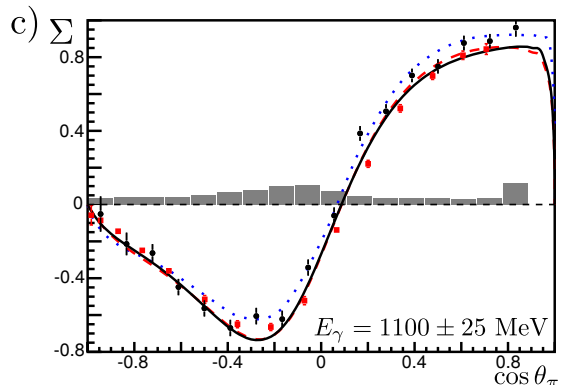

Figure 2. (Color online) a) A typical $\phi_{\pi}$-distribution with a fit using eq. (2). b,c) The beam asymmetry $\Sigma$ as a function of $\cos \theta_{\pi}$ for $E_{\gamma}=800 \mathrm{MeV}$ and for $E_{\gamma}=1100 \mathrm{MeV}$. Black dots show our data, red squares GRAAL data 20]. The curves represent predictions from different partial wave analyses. Solid (black) curve: BnGa 24]; dashed (red): SAID [22]; long-dashed (black): BnGa with $E_{0^{+}}$and $E_{2^{-}}$amplitudes from SAID; dotted (blue): MAID [23]; dashed-dotted (black): BnGa with $E_{0^{+}}$ and $E_{2}$ - amplitudes from MAID. Gray area shows the systematic error due to interactions on nuclei and uncertainty in the photon polarization.

forward calorimeter crystals. Additionally all events are removed, if the calculated beam photon energy (under the assumption that the reaction occurred on a proton) falls below the tagging threshold. With these cuts the reconstruction efficiency for reaction (11) on free protons in the butanol target exceeds $60 \%$.

Fig. 10 shows the resulting distribution of missing masses for events passing the selection criteria, with butanol, carbon, or hydrogen as target material. By proper scaling, the sum of distributions from hydrogen and carbon reproduces very well the distribution obtained with the butanol target. The missing mass distributions show a peak at the proton mass and a tail due to events where an additional meson was produced but escaped detection. A final cut is now applied on the missing mass at $m_{p} \pm 50 \mathrm{MeV}$. The resulting $\cos \theta_{\pi}$ distribution of these events is exhibited in fig. 11. Again, the sum of distributions from hydrogen and carbon match the distribution obtained with the butanol target; there is no new scaling factor involved.

For the double-polarization observable $G$ the correction factor for reactions on nuclei is the dilution factor, displayed in fig. 1 1 . Due to the kinematic cuts, the dilution factor depends on both the photon energy $E_{\gamma}$ and the $\pi^{0}$ scattering angle $\theta_{\pi}$. For a backward $\pi^{0}$, the proton momentum is high and therefore detected in the crystals with a high efficiency.

A typical distribution of the measured $\phi_{\pi}$-dependent data yields with a fit using eq. (2) is shown in fig. 22. Obviously, there is a large $\cos 2 \phi_{\pi}$ contribution but the data are not symmetric with respect to $\phi_{\pi}=0$ : there is a significant $\sin 2 \phi_{\pi}$ contribution from which the desired observable $G$ is deduced. Fig. 2b,c show the beam asymmetry $\Sigma$ extracted from this and other similar plots for two energy bins. The systematic error due to reactions on nuclei are shown as gray bars. The new $\Sigma$ data are in excellent agreement with earlier measurements of GRAAL [20].

The observable $G$ is determined from eq. (2), as well

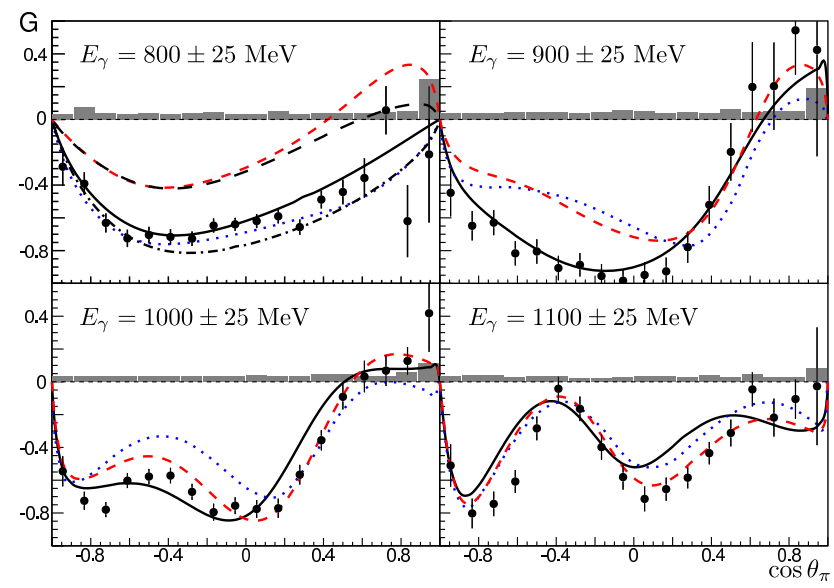

Figure 3. (Color online) The polarization observable $G$ as a function of $\cos \theta_{\pi}$ from $E_{\gamma}=800 \mathrm{MeV}$ up to $E_{\gamma}=1100 \mathrm{MeV}$. Systematic errors are shown in gray bars. Curves: see fig. 2

as by reversing the target polarization, or changing the photon polarization plane by $\pm \pi / 4$. The results are consistent, their spread is used to determine the systematic errors. These include the systematic uncertainty in the dilution factor, errors due to background in $\cos \theta_{\pi}>0.9$, the relative uncertainty in the target polarization of $\pm 2 \%$, and the relative uncertainty in the photon polarization of $\pm 5 \%$ 21]. Fig. 3 shows the angular distribution of the polarization observable $G$ for selected photon energy ranges. The error bars give the statistical errors, the systematic errors are shown as gray bars.

The new data on $\Sigma$ and $G$ are compared to the predictions from the SAID (SN11) 22], MAID [23], and BnGa 24] partial wave analyses. The results are surprising.

Already at rather low energies, in the region of the four-star resonances $N(1440), N(1535)$ and $N(1520)$, very significant differences in the predictions can be observed (see figs. 22 and 3). At 800 and $900 \mathrm{MeV}$, the SAID prediction is incompatible with the data on $\Sigma$ and $G$, MAID disagrees with the data on $\Sigma$ and on $G$ at 


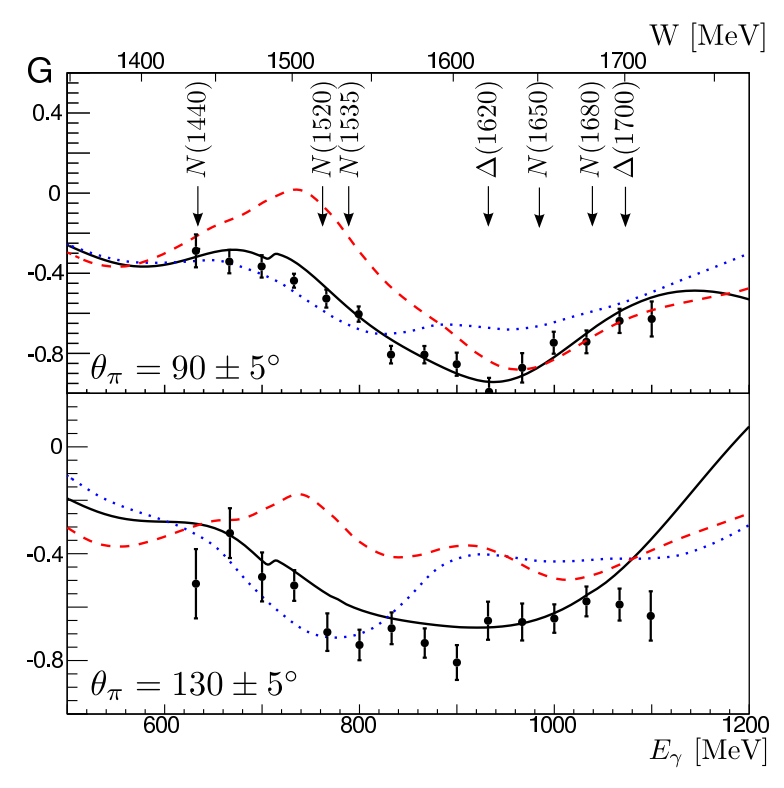

Figure 4. The double-polarization observable $G$ as a function of energy for two selected bins in $\theta_{\pi}$. Curves: see fig. 2, For comparison the positions of several resonances are marked.

$900 \mathrm{MeV}$. At 1000 and $1100 \mathrm{MeV}$, BnGa, MAID and SAID give a reasonable description, even though MAID is higher at $1000 \mathrm{MeV}$. These conclusions are supported by fig. 4 where $G$ is shown as a function of $E_{\gamma}$ for two scattering angles and compared to PWA predictions. At low energies, SAID deviates strongly from the data; in the $900-1000 \mathrm{MeV}$ range, the consistency between data and MAID or SAID predictions is poor. Above, none of the partial wave analyses reproduces the data.

We have studied possible reasons for the discrepant results. In this discussion, the two electric multipoles $E_{0^{+}}$ and $E_{2-}$ play a leading role. If we replace the $\mathrm{BnGa} E_{0+}$ and $E_{2^{-}}$multipoles by the corresponding SAID multipoles, we reproduce the SAID results for $\Sigma$ and $G$ over a wide energy range, and the same observation holds true for MAID. The reason for the deviation between data and MAID and SAID is obviously traced to these two multipoles. All other multipoles can be exchanged without a large impact.

Resonances with no angular momentum between proton and $\pi^{0}\left(l_{\pi}=0\right)$ contribute to the $E_{0^{+}}$multipole which is sensitive to the $N(1535), \Delta(1620)$ and $N(1650)$ resonances. The resonances $N(1520)$ and $\Delta(1700)$ with $l_{\pi}=2$ and total spin $J=3 / 2$ contribute to the $E_{2^{-}}$ multipole. Indeed, the three partial wave analyses BnGa, MAID, and SAID give significantly different helicity amplitudes for these resonances, in particular for the $A_{1 / 2}$ amplitude of $N(1535)$ and for $A_{3 / 2}$ of $N(1520)$ (see Table 【).

In the medium energy range, in the third resonance region, the $\gamma p \rightarrow p \pi^{0}$ cross section is dominated by the $J^{P}=5 / 2^{+} N(1680)$ resonance. In this regime, there is

\begin{tabular}{|c|c|c|c|c|}
\hline $\boldsymbol{E}_{\mathbf{0}}+$ & $N(1535) 1 / 2^{-}$ & $N(1650) 1 / 2^{-}$ & $\Delta(1620) 1 / 2^{-}$ & \\
\hline Solution & $A^{1 / 2}$ & $A^{1 / 2}$ & $A^{1 / 2}$ & \\
\hline BG2011-02 & $105 \pm 10$ & $33 \pm 7$ & $52 \pm 5$ & \\
MAID-2007 & 66 & 33 & 66 & \\
SAID-2011 & $99 \pm 2$ & $65 \pm 25$ & $64 \pm 2$ & \\
\hline \hline $\boldsymbol{E}_{\mathbf{2}-}$ & \multicolumn{2}{|c|}{$N(1520) 3 / 2^{-}$} & \multicolumn{2}{|c|}{$\Delta(1700) 3 / 2^{-}$} \\
\hline Solution & $A^{1 / 2}$ & $A^{3 / 2}$ & $A^{1 / 2}$ & $A^{3 / 2}$ \\
\hline BG2011-02 & $-22 \pm 4$ & $131 \pm 10$ & $160 \pm 20$ & $165 \pm 25$ \\
MAID-2007 & -27 & 161 & 226 & 210 \\
SAID-2011 & $-16 \pm 2$ & $156 \pm 2$ & $109 \pm 4$ & $84 \pm 2$ \\
\hline
\end{tabular}

Table I. Helicity amplitudes of low-lying negative-parity $N$ and $\Delta$ resonances contributing to the $E_{0^{+}}$and $E_{2^{-}}$multipoles (in $\mathrm{GeV}^{-\frac{1}{2}} 10^{-3}$ ).

good agreement between the three predictions. The properties of this resonance are obviously well defined: For the leading $A_{3 / 2}$ helicity amplitude, BnGa finds $135 \pm 6$, MAID 134, and SAID $141 \pm 3$ (in $\mathrm{GeV}^{-\frac{1}{2}} 10^{-3}$ ).

In the high energy region, increasing differences between the predictions can be observed for some angles. This is the so-called fourth resonance region where many resonances exist with questionable evidence for their existence and, if they exist at all, with at least poorly defined properties. New data on $G$ in this energy region will help to disentangle the spectrum of nucleon resonances [21].

Summarizing, we have reported the first measurement of the double-polarization observable $G$ in the reaction $\vec{\gamma} \vec{p} \rightarrow p \pi^{0}$ in a wide range of energies and covering the full solid angle. The new data on $G$ resolve the discrepant results on the helicity amplitudes of low-lying four-star nucleon resonances obtained from BnGa, MAID, and SAID and are an important step towards a complete data base which will define unambiguously the nucleon excitation spectrum.

We thank the technical staff of ELSA and the participating institutions for their invaluable contributions to the success of the experiment. We acknowledge support from the Deutsche Forschungsgemeinschaft (SFB/TR16) and Schweizerischer Nationalfonds.

[1] M. Gell-Mann, Phys. Rev. 125, 1067 (1962).

[2] G. Zweig, CERN-TH-401 (1964).

[3] H. Fritzsch, P. Minkowski, Nuovo Cim. A30, 393 (1975).

[4] R. G. Edwards et al., Phys. Rev. D84, 074508 (2011).

[5] N. Isgur, G. Karl, Phys. Rev. D18, 4187 (1978).

[6] U. Löring et al., Eur. Phys. J. A10, 395 (2001).

[7] K. M. Watson, Phys. Rev. 182, 228 (1954).

[8] R. Beck et al., Phys. Rev. Lett. 78, 606 (1997).

[9] G. Blanpied et al., Phys. Rev. Lett. 79, 4337 (1997).

[10] A. S. Omelaenko, Yad. Fiz. 34, 730 (1981).

[11] Y. Wunderlich, Diploma Thesis, Univ. Bonn (2012).

[12] W. -T. Chiang, F. Tabakin, Phys. Rev. C55, 2054 (1997).

[13] A. M. Sandorfi et al., J. Phys. G 38, 053001 (2011). 
[14] W. Hillert, Eur. Phys. J. A28S1, 139 (2006).

[15] D. Elsner et al., Eur. Phys. J. A39, 373 (2009).

[16] H. Dutz et al., Phys. Rev. Lett. 93, 032003 (2004).

[17] A. Thiel, PhD Thesis, Universität Bonn (2012).

[18] G. Suft et al., NIM A538, 416 (2005).

[19] V. Crede et al., Phys. Rev. C84, 055203 (2011).
[20] O. Bartalini et al., Eur. Phys. J. A26 399 (2005).

[21] A. Thiel, H. Eberhardt et al., publication in preparation.

[22] R. Workman et al. Phys. Rev. C85, 025201 (2012).

[23] D. Drechsel et al., Nucl. Phys. A645, 145 (1999).

[24] A. V. Anisovich et al., Eur. Phys. J. A48, 15 (2012). 